\section{Relación entre nivel de conocimiento y manejo de los residuos biocontaminados, y contaminación generada en dos clínicas odontológicas universitarias}

Relation between level of knowledge and waste management of biosanitary, and pollution generated in two university dental clinics

\section{Resumen}

El propósito fue relacionar el nivel de conocimientos con el manejo de los residuos biocontaminados y determinar el nivel de contaminación generados en las clínicas odontológicas de la Facultad de Odontología de la Universidad Nacional Mayor de San Marcos (UNMSM) y de la Universidad Nacional Federico Villarreal (UNFV). El tipo de estudio fue transversal analítico. Participaron 299 personas entre estudiantes, docentes y personal de mantenimiento .Se utilizó una encuesta, ficha de observación y análisis microbiológico. Se encontró un nivel de conocimiento del personal sobre el Manejo de Residuos Biocontaminados bajo y muy bajo. No existieron diferencias de género en el personal de UNMSM $(\mathrm{p}=$ 0.55), mientras que en la UNFV, el sexo masculino presentó un menor conocimiento $(\mathrm{p}=0.038)$. El Manejo de residuos contaminados biosanitarios y anatomopatologicos desde su generación hasta su disposición final es inadecuado (UNMSM $92.3 \%$ y 58,5 \%; UNFV $100.0 \%$ y 51,8 \% respectivamente). El manejo de punzocortantes es adecuado (UNMSM $77.4 \%$, UNFV 100\%). Se hallaron bacterias mesofilas, heterotróficas y hongos dentro de los ambientes. En dos escupideras, se halló Pseudomonas y colonias Lactosa+. El nivel de conocimiento estuvo relacionado con el manejo inadecuado de los residuos biologicos contaminados, biosanitarios y anatomopatológicos, mientras que no guardó relación con el manejo de los residuos punzocortantes. Existe contaminación biológica en los ambientes estudiados de las clínicas. No se cumple con las normas técnicas del Ministerio de Salud; a excepción del manejo de los residuos punzocortantes.

Palabras clave: Residuos biocontaminados, residuos peligrosos, servicios odontológicos, infecciones, contaminación.

\begin{abstract}
The purpose was to relate the level of knowledge, biocontaminated waste management. and the level of pollution generated in two Dental Clinics: Clinicof the Faculty of Dentistry at the National University of San Marcos (San Marcos) and the Universidad Nacional Federico Villarreal (UNFV). The type of study was cross-sectional 1 . Attended by 299 people including students, teachers and maintenance staff. Was used an observation sheet and microbiological analysis. There was a low and very low level of staff knowledge about biocontaminated Waste Management. There were no gender differences in UNMSM staff ( $\mathrm{p}=0.55)$, while in UNFV, males showed less knowledge $(\mathrm{p}=0.038)$. The biocontaminated and pathologic waste management from generation to final disposal is inadequate (UNMSM $92.3 \%$ and $58.5 \%, 100.0 \%$ and $51.8 \%$ UNFV respectively). The handling of needles is adequate $(77.4 \%$ UNMSM, UNFV $100 \%$ ). Were found mesophilic bacteria, heterotrophic bacteria and fungi within environments. In two spittoons were found Lactose + and Pseudomonas colonies. The level of knowledge was associated with improper management of contaminated biological waste, while was not related to the handling of sharps waste. It was concluded that exists relation between level of staff knowledge and inadequate management of biological and pathological waste. There is biological contamination in the studied environments clinics and lack of compliance with the technical standards of the Ministry of Health, with exception of needles waste.
\end{abstract}

Key words: biocontaminated waste, biosanitary waste, dental, infections, pollution.

Artículo Original

\section{Sylvia Chein Villacampa ${ }^{1}$, Carlos Campodónico \\ Reátegui', Lourdes Benavente Lipa', Elmo Palacios Alva ${ }^{1}$, María Alvarez Paúcar ${ }^{1}$, Teresa Evaristo Chiyong', María Ventocilla Huasupoma ${ }^{1}$, Marco Tulio Madrid Chumacero ${ }^{1}$, Andres Pachas Vásquez², María del Carmen Huapaya- Pardavé ${ }^{2}$}

1 Docentes de la Facultad de Odontología, Universidad Nacional Mayor de San Marcos.

2 Estudiante Pre grado de la Facultad de Odontología, Universidad Nacional Mayor de San Marcos.

\section{Correspondencia:}

Esp. Sylvia Chein Villacampa

Facultad de Odontología. Av. Germán Amezaga s/n, Lima, 1 Perú.

Teléfono: 6197000 anexo 3402

Correo electrónico: sychevi7720@hotmail.com
Fecha de recepción: 19-07-12

Fecha de aceptación: 25-09-12 


\section{Introducción}

La contaminación ambiental figura entre las principales preocupaciones de la sociedad mundial debido a los efectos que producen en la salud. La generación y manejo de los residuos sólidos es uno de los problemas ambientales más serio. ${ }^{1}$ Actualmente muchos países preocupados por el deterioro de la salud a nivel mundial se han dado la tarea de establecer programas de bioseguridad en el sector salud. En Odontología que es considerada una profesión de alto riesgo, por el carácter médico de los actos que a diario realiza, existe preocupación por la salubridad de nuestros ambientes de trabajo e instalaciones, así como la preservación de la salud de nuestros pacientes, profesionales y personal auxiliar. En nuestro país existen muy pocos estudios a nivel nacional que reportan el manejo de residuos biocontaminados y su relación con los efectos sobre la salud en poblaciones expuestas, y los que existen concluyen que se manejan inadecuadamente en los establecimientos de salud, lo que constituye un factor de riesgo para la trasmisión de enfermedades, para la población que atiende o labora, aledaña y el medio ambiente. ${ }^{2,3,4,5,6,7,8,9,10}$ Las Facultades de Odontología son centros de formación de recursos humanos que prestan servicios de salud a la comunidad y es su responsabilidad prevenir las enfermedades y contribuir a minimizar el riesgo ambiental, de la misma manera que las demás instituciones prestadoras de servicios de salud. La práctica de la odontología implica la generación de residuos biocontaminados, especiales y comunes. Los residuos que contienen diversidad de material biológico potencialmente nocivo como: bacterias, virus, microorganismos, toxinas, sangre, saliva, fluidos y otros materiales y sustancias capaces de dańar el medio ambiente y la salud, si su manejo es inadecuado, podrían provocar enfermedades si llegaran a los basureros municipales mezclados con gasas, sangre, jeringas, algodones, restos de medicamentos y otros que salen de quirófanos y salas de atención a pacientes. ${ }^{2,4,10,15,16,17} \mathrm{El}$ propósito del estudio fue relacionar el nivel de conocimiento con el manejo de los residuos biocontaminados y determinar el nivel de contaminación generada en los servicios odontológicos de Lima 2011, para luego establecer e implementar un sistema de una gestión de los residuos sólidos ${ }^{11,12,13,14}$ como lo tienen otras establecimientos de salud.
Tabla 1. Personal que labora en los servicios odontológicos según condición laboral e Institución de procedencia. Lima 2011.

\begin{tabular}{lrrrr}
\hline & Condición & \multicolumn{2}{c}{ Institución de procedencia } & \multicolumn{1}{c}{ Total } \\
\cline { 2 - 3 } & \multicolumn{1}{l}{ UNMSM } & UNFV & 53 & 218 \\
Estudiante & 165 & $56.4 \%$ & $72.9 \%$ \\
& $80.5 \%$ & 30 & 60 \\
Docente & 30 & $31.9 \%$ & $20.1 \%$ \\
& $14.6 \%$ & 11 & 21 \\
Personal de Limpieza & 10 & $11.7 \%$ & $7.0 \%$ \\
& Total & $4.9 \%$ & 94 & 299 \\
& 205 & $100.0 \%$ & $100.0 \%$ \\
\hline \hline
\end{tabular}

\section{Material y métodos}

El estudio es transversal analítico. La muestra estuvo conformada por 299 personas entre estudiantes, docentes y personal de mantenimiento y los servicios odontológicos ( $\mathrm{SO}$ ) de las $\mathrm{Fa}$ cultades de Odontología de la Universidad Nacional Mayor de San Marcos (UNMSM) y la Universidad Nacional Federico Villarreal (UNFV). La muestra estuvo conformada por personas de ambos sexos, cuya institución de procedencia fue la Universidad Nacional Mayor de San Marcos (UNMSM) y Universidad Nacional Federico Villarreal (UNFV). En un $72,9 \%$ por estudiantes, 20,1\% docentes y $7 \%$ personal de limpieza. Ver Tabla 1.

Las técnicas de recolección de información empleadas fueron: encuesta, fichas de observación y análisis microbiológico. Mediante el cuestionario se

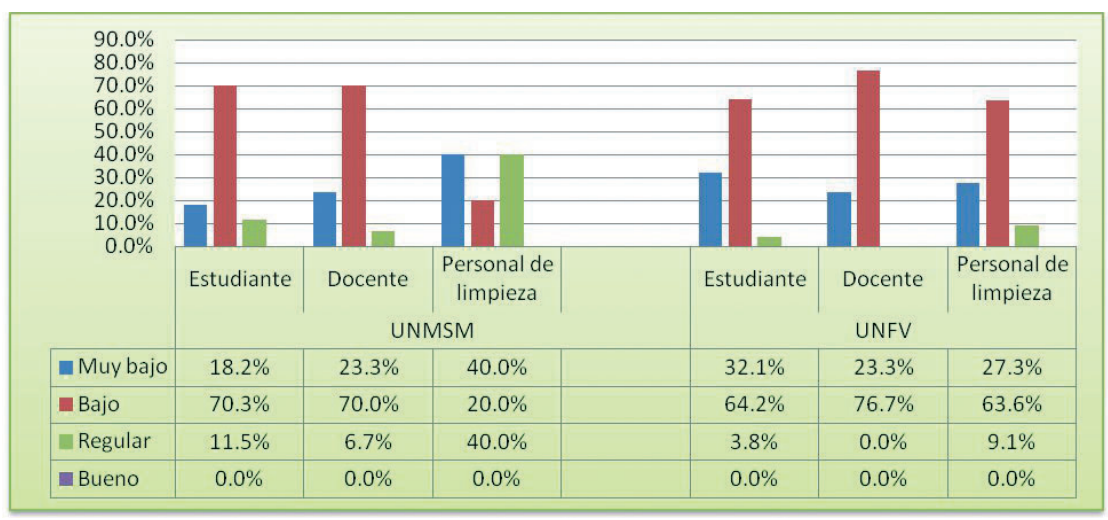

Fig 1. Nivel de conocimiento según condición laboral e institución de procedencia. Lima 2011

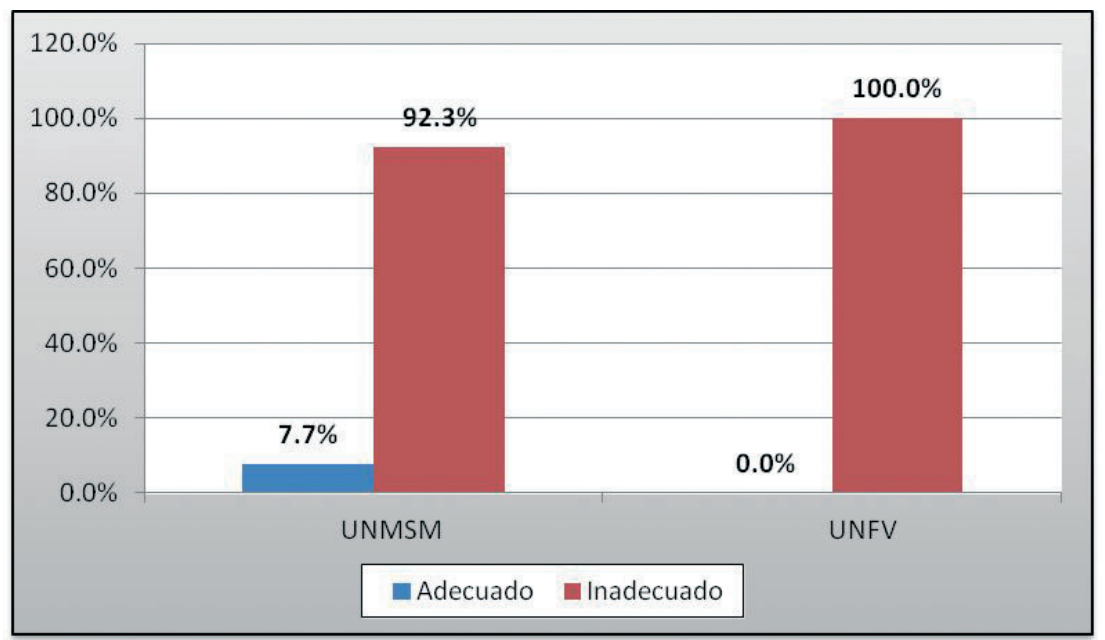

Fig 2. Manejo de desechos bilógicos infecciosos: Biosanitarios por estudiantes y docentes según institución de procedencia. Lima 2011 
determinó el nivel de conocimientos en el manejo de los residuos biocontaminados del personal que labora en las clínicas odontológicas de las Facultades de Odontología de la UNMSM y UNFV, en las fichas de observación se registraron in situ el manejo de los residuos biológicos desde su generación hasta sus disposición final y para el análisis microbiológico se consideró: aire ambiental, superficies y equipos; la toma de las muestras y la marcha técnica de este proceso se realizaron según lo establecido en las normas del Laboratorio Nacional de Infecciones Nosocomiales, ${ }^{18}$ empleando los medios de cultivo Agar Sangre (TSA), Agar McConkey y Agar Sabouraud Glucosado. Para el análisis estadístico se utilizo SPSS 15 , mediante las pruebas de Chi cuadrado y de Spearman.

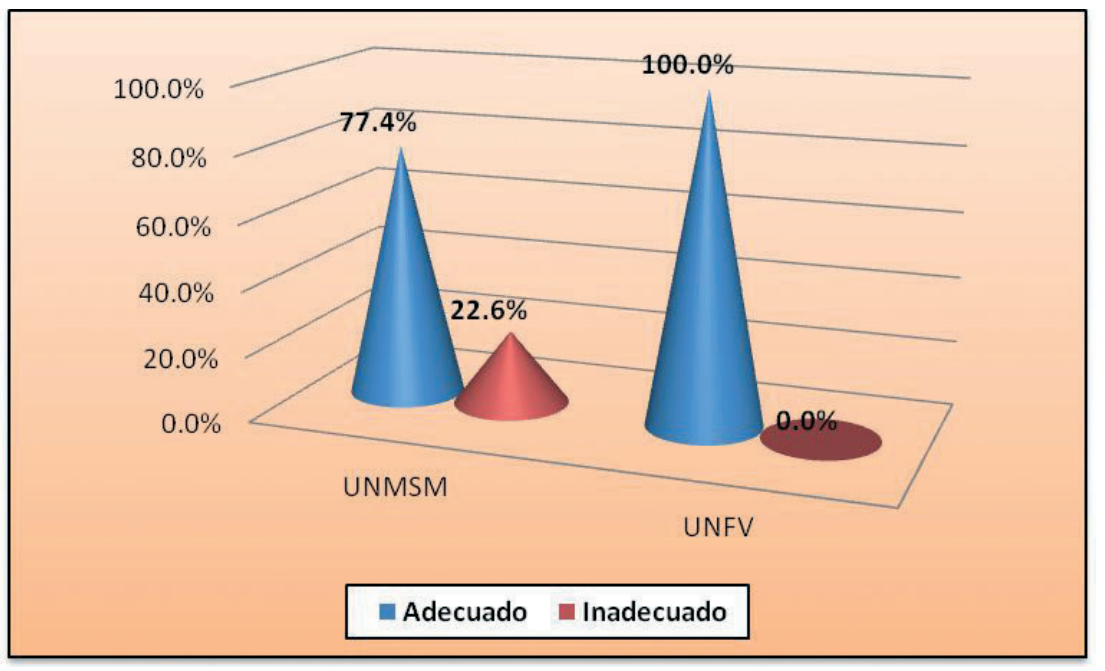

Fig 3. Manejo de desechos biológicos infecciosos punzocortantes por estudiantes y docentes según institución de procedencia. Lima 2011.

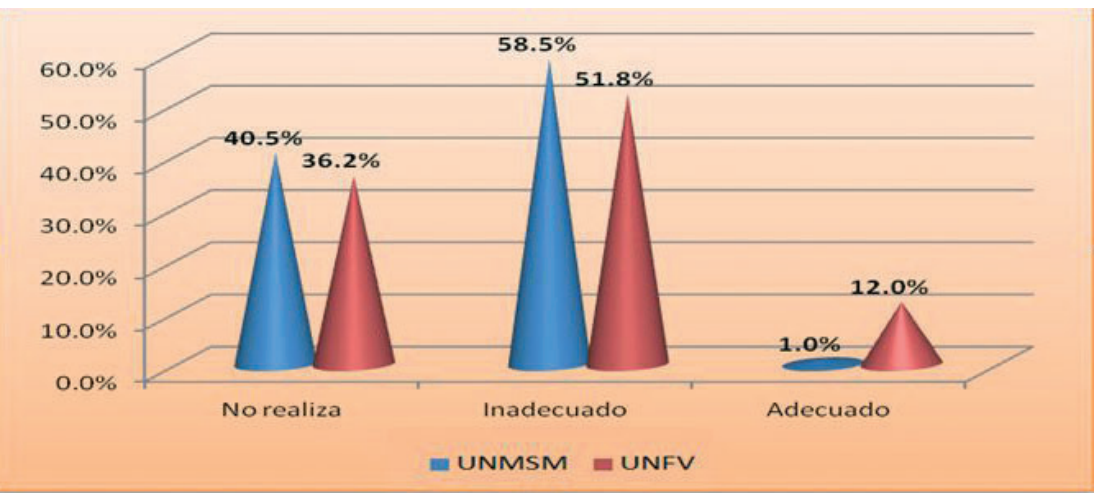

Fig 4. Manejo de desechos biológicos infecciosos anatomopatológicos por estudiantes y docentes según institución de procedencia. Lima 2011

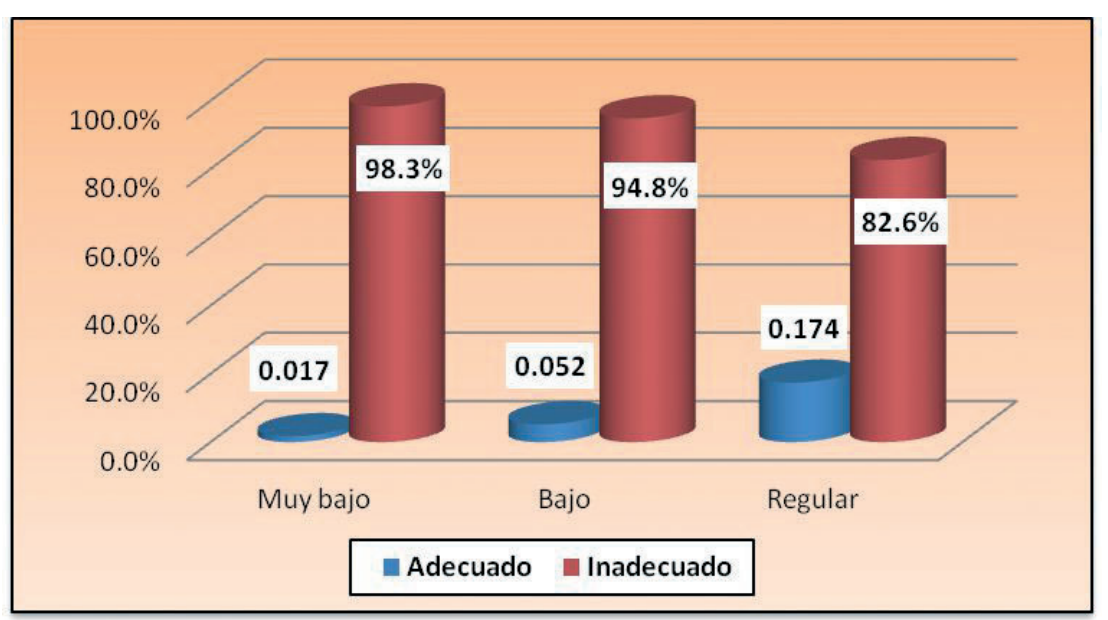

Fig 5. Manejo de desechos biológicos infecciosos biosanitarios según nivel de conocimiento. Lima 2011

\section{Resultados}

Se observa un nivel de conocimiento de bajo a muy bajo sobre manejo de residuos biocontaminados del personal que labora en las clínicas odontológicas de ambas instituciones. No existe el conocimiento bueno. Ver Fig 1.

El nivel de conocimiento que tienen las personas del sexo masculino y femenino en la UNMSM no mostraron diferencias $(p=0.55)$, mientras que en la UNFV si se observa esa diferencia presentándose un menor conocimiento en el sexo masculino $(\mathrm{p}=0.038)$.

Se observa que el manejo de residuos biosanitarios es inadecuado (UNMSM 92.3 y UNFV 100.0\%) en ambas instituciones observándose mayormente ese efecto en la UNFV. Se observa una diferencia estadísticamente significativa entre las instituciones para el manejo de residuos biosanitarios ( $\mathrm{p}=0.004)$. Ver Fig 2 .

Sobre el manejo de residuos punzocortantes observamos que en ambas instituciones lo hacen mayormente en forma adecuada (UNMSM 77.4 $\%$, UNFV $100 \%$ ) observándose una diferencia en UNFV. Se observa una diferencia estadísticamente significativa entre las instituciones para el manejo de desechos punzocortantes. $(\mathrm{p}=0.000)$. Ver Fig 3.

Se apreció un predomino del manejo inadecuado de los residuos anatomopatológico en ambas instituciones (UNMSM 58,5 \%, UNFV $51.8 \%$ ). Se encuentra diferencia estadísticamente significativa entre las instituciones para el manejo de desechos Anatomopatológico $(\mathrm{p}=0.34)$. Ver Fig 4 .

En cuanto al nivel de conocimientos que es bajo y muy bajo, este guarda relación con el manejo inadecuado de los residuos biosanitarios en la muestra total. Existe asociación estadísticamente significativas entre el nivel de conocimientos y el manejo de residuos biosanitarios (Chi cuadrado $8.19 \mathrm{p}=$ $0.017)$, predominando el nivel de conocimiento bajo en el manejo incorrecto de los residuos.

Se observó en la muestra total que el nivel de conocimiento no guarda relación con el manejo adecuado de los residuos punzocortantes $(\mathrm{p}=0.060)$. Ver Fig 6.

Sin embargo, el nivel de conocimiento guardó relación con el manejo inadecuado de los residuos anatomopatológicos. No existe asociación estadísticamente significativa entre el nivel de conoci- 


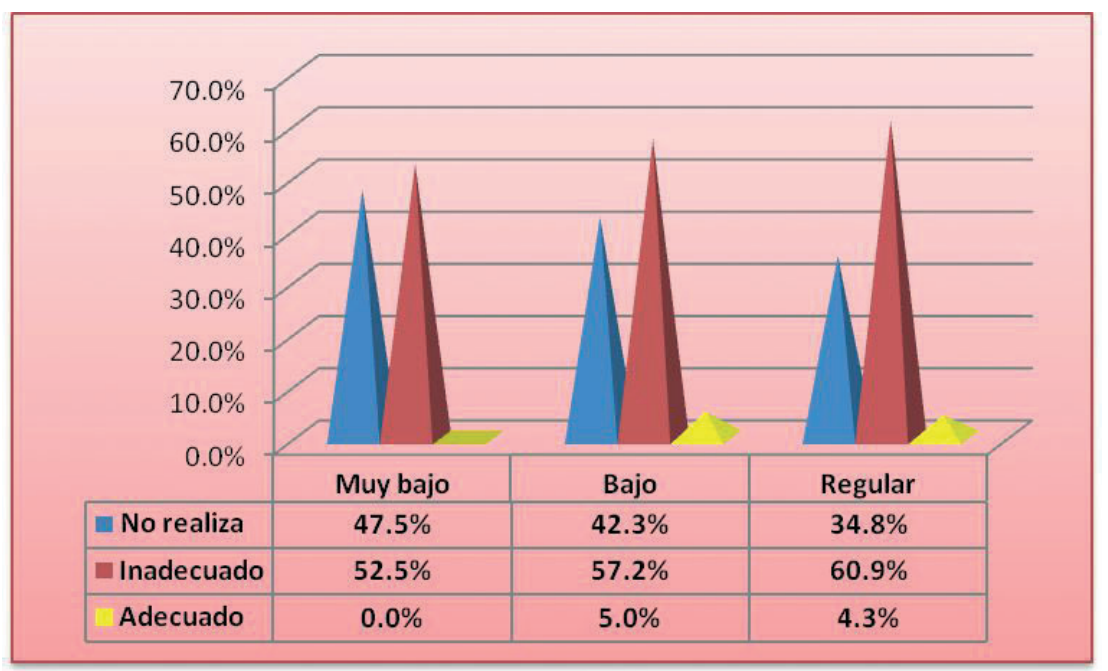

Fig 6: Manejo de desechos biológicos infecciosos punzocortantes según nivel de conocimiento. Lima 2011

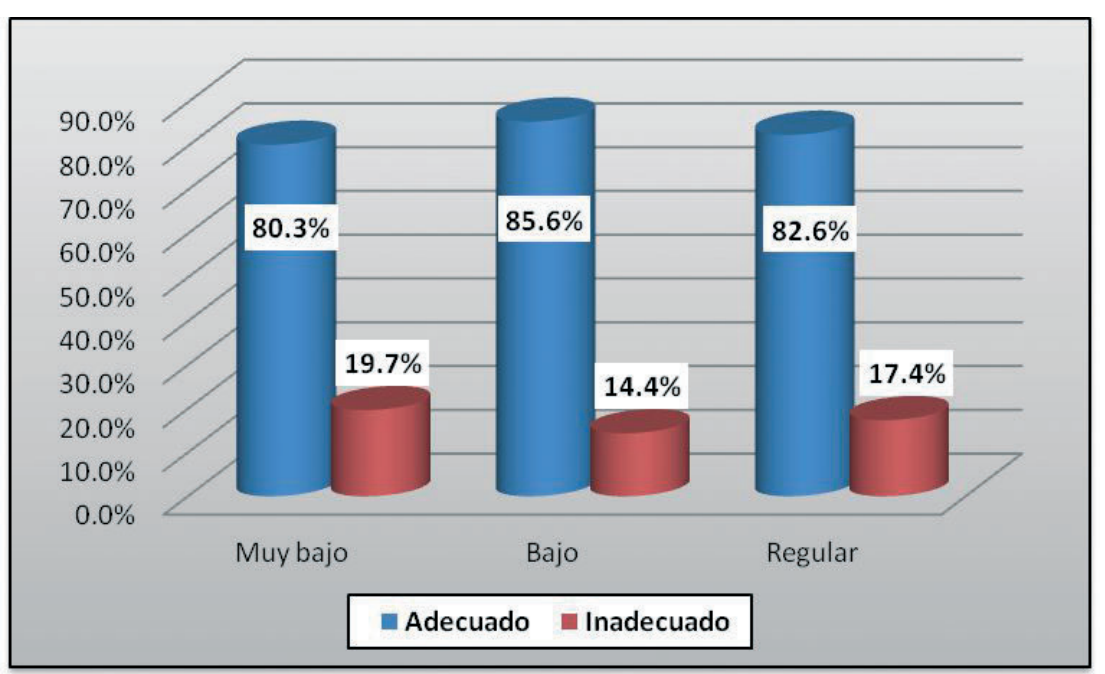

Fig 7. Manejo de desechos biológicos infecciosos anatomopatológico según nivel de conocimiento. Lima 2011

miento y el manejo de desechos anatomopatológico. $(\mathrm{p}=0.22)$. Ver Fig 7 .

La mayoría de las etapas del manejo de los desechos de los servicios odontológicos de ambas instituciones no cumplen con los pasos establecidos en cada una de ellas, según la Norma Técnica del MINSA.

Se encontraron en los lugares/recipientes monitoreados bacterias mesofilas y heterotróficas lo cual es frecuente, sin embargo se destaca el hallazgo de Pseudomonas en una de las escupideras analizadas (UNMSM), y coliformes en otra (UNFV).

\section{Discusión}

De acuerdo a la revisión bibliográfica, se debe indicar que hay muy pocos estudios sobre manejos de residuos sólidos y de residuos producidos en los centros de atención odontológica. ción al manejo de los residuos biocontaminados, este se presenta en un nivel de bajo a muy bajo.

Se observa que el manejo de los residuos biosanitarios es inadecuado (UNMSM 92.3 y UNFV $100.0 \%$ ) en ambas instituciones observándose mayormente ese efecto en la UNFV. Sin embargo, respecto al manejo de residuos punzocortantes observamos que en ambas instituciones se da en forma adecuada (UNMSM 77.4 \%, UNFV $100 \%$ ) estos resultados son similares a los encontrados por Montaño $\mathrm{ML}(2006)^{16}$; anatomopatológicos es inadecuado en ambas instituciones (UNMSM 58,5\%, UNFV $51.8 \%$ ).

Encontramos que existe relación directa entre el nivel de conocimientos y el manejo de residuos biosanitarios y anatomopatologicos. Asimismo, que no existe relación entre el nivel de conocimiento bajo y muy bajo y manejo adecuado de los residuos punzocortantes (agujas, bisturíes, etc) de parte de los estudiantes y docentes, lo que podría deberse a que los consideran elementos de alto riesgo para su salud.

En el manejo de los residuos se observa en la mayoría de las etapas que no se cumple con los pasos establecidos según la Norma Técnica del Ministerio de Salud, ${ }^{14}$ lo que constituye un peligro potencial de infección y contaminación para los estomatólogos, el personal de mantenimiento y la sociedad en general, resultados similares encontraron Gómez R $(2004)^{2}$; Manrique $\mathrm{L}(2004)^{3}$; Velasco A, Campodónico $\mathrm{C}(2005)^{4}$; Mérida E(2006) ${ }^{6}$; Correa L y col(2007) ${ }^{7}$; López $\mathrm{R}^{9}$; Conde $\mathrm{C}^{10}$; La Corte $\mathrm{E}^{15}$

Śco el estudio en los servicios odonfacultades de odont logía de UNMSM y UNFV donde se evaluó al personal que labora en ellos: estudiantes, docentes y personal de limpieza, para determinar su nivel de conocimientos y el manejo de los residuos biocontaminados, en los resultados obtenidos se evidencia desconocimiento sobre su manejo y de las normas internacionales y nacionales, resultados similares a los de Gómez $\mathrm{R}(2004)^{2}$; Manrique L(2004) ${ }^{3}$; Velasco A, Campodónico $\mathrm{C}(2005)^{4}$; Sirit $\mathrm{Y}(2005)^{5}$; Mérida $\mathrm{E}(2006)^{6}$; Correa L y $\operatorname{col}(2007)^{7}$; Rubio $\mathrm{M}(2008)^{8}$; López $\mathrm{R}^{9}$; Conde $\mathrm{C}^{10}$; La Corte $\mathrm{E}^{15}$, pero difieren con Rivera $\mathrm{E}(2002)$ quien encontró que un alto porcentaje de internos tenía conocimiento y cumplían con el manejo correcto de desechos contaminados.

Se observa en ambas instituciones (UNMSM, UNFV) que no existe un nivel de conocimiento bueno en rela-
Para determinar el nivel de contaminación biológica de los servicios odontológicos de las dos universidades nacionales, se utilizo diferentes técnicas y muestras, los resultados obtenidos demuestran datos lógicos y esperados y otros no esperados, que pueden tener algunas explicaciones. La diferencia de presencia y recuento microbiano entre ambas muestras (la inicial y la final) nos indica el grado de contaminación de cada uno de los lugares monitoreados, ya que no existen en nuestro país normas, estándares de calidad microbiológica o límites máximos permisibles, de los recipientes y/o lugares monitoreados en este estudio. Por lo tanto nuestros resultados no tienen parámetros de referencia para conocer el grado de contaminación biológica, sin embargo nuestra medida de la contaminación ha sido establecida de forma cualitativa y 
cuantitativa comparando los resultados entre las muestras iniciales y las finales; así como los resultados entre una muestra y otra. Debe destacarse el hallazgo de Pseudomonas en una de las escupideras analizadas, por la peligrosidad que esta bacteria representa. Asimismo, es destacable positivamente mencionar que no se encontraron coliformes, que son indicadores de contaminación fecal, excepto en la muestra final de la escupidera del servicio odontológico de una de las universidades que se encontró en el Agar Mac Conckey colonias lactosa + , sin embargo no se llegó a la identificación final si eran o no bacterias del grupo coliformes. El hecho de encontrar en los lugares/recipientes monitoreados bacterias mesofilas y heterotróficas es frecuente.

\section{Conclusiones}

1. El nivel de conocimientos del personal que labora en los servicios odontológicos es bajo y muy bajo y se asocia con el manejo inadecuado de los residuos biocontaminados desde su generación hasta su disposición final.

2. No se cumple con las normas oficiales de bioseguridad; a excepción del manejo de los desechos punzocortantes.

3. Existe contaminación biológica en los lugares monitoreados de los Servicios Odontológicos.

4. Se considera necesario una serie de medidas correctivas respecto como incluir contenidos en las asignaturas de las Facultades de las Ciencias de la Salud sobre el manejo de residuos generados en los Establecimientos de Salud y Servicios Médicos de Apoyo; difundir y efectivizar el cumplimiento de las Normas Técnicas del MINSA para el manejo de residuos en los establecimientos de salud y dictar normas, estándares de calidad microbiológica; elaborar protocolo de manejo de desechos y capacitar el personal de salud y de limpieza sobre el proceso de gestión de los residuos generados en los establecimientos de salud, tomando en cuenta y destacando los peligros de su mal manejo.
Agradecimiento. Al Vicerrectorado de Investigación de la Universidad Nacional Mayor de San Marcos por el apoyo económico que nos brindó para la ejecución del presente estudio.

\section{Referencias Bibliográficas}

1. CancharI G y Ortiz O. Valorización de los residuos sólidos en la ciudad universitaria de la Universidad $\mathrm{Na}$ cional Mayor de San Marcos. Rev. Inst. Investig. Fac. Minas Metal Cienc. Geogr. 2008; 11(21):95-99.

2. Gómez R El manejo de residuos peligrosos biológico-infecciosos en los consultorios dentales. Estudio de campo. Rev ADM 2004; LXI(4):137-141

3. Manrique L y col. Manejo de desechos peligrosos en los establecimientos de salud del área Alajuela oeste. Rev. costarric. salud pública. 2004;13(24):75-81.

4. Velazco A, Campodónico C. Bioseguridad en el manejo y eliminación de residuos en los centros de atención odontológica del cono norte de Lima Metropolitana 2005. Odontología Sanmarquina 2009; 12(2):70-73

5. Sirit Y, Matos J, Panunzio A, Nuñez M, Bellorín M. Desechos Biológicos Generados en Laboratorios de la Facultad de Medicina de una Institución Universitaria. Revista Kasmera. 2005; 33 (1):

6. Mérida E. Los efectos del incumplimiento reglamentario en cuanto al manejo de desechos sólidos hospitalarios Guatemala 2006. Consultado 23-10-2011. Disponible en: http://biblioteca.usac.edu.gt/ tesis/04/04_5933.pdf

7. Correa LB, Lunardi VL y De Conto SM. El proceso de formación en salud: El tema residuos solidos de servicios de salud en vivencias prácticas. Rev. Bras. Enferm. 2007;60(1):21-25.

8. Rubio MO, Avila GA y Gomez BA. Actitudes de estudiantes de Enfermería Mexicanos al manejar residuos peligrosos biologico infecciosos. Rev.Redalyc.2008; 12(3):479-484.
9. López RA Estudio de generación de residuos peligrosos biológico infecciosos en centros de atención medica en el estado de Oaxaca. Consultado 28-11-2011. Disponible en: http:// bvsde.per.paho.org/bvsaidis/resisoli/ mexicona/R-0174.pdf

10. Conde $\mathrm{C}$ y et. al Manejo de desechos biológico-infecciosos en las clínicas periféricas de la FESI. Coloquio XVII Facultad de Estudios Superiores Iztacala.

11. Ley No 27314, Ley General de Residuos Sólidos aprobada el 21 de julio del 2000.

12. Reglamento de la Ley $\mathrm{N}^{\circ} 27314$, aprobado mediante Decreto Supremo $\mathrm{N}^{\circ}$ 057-2004- PCM el 24 de julio del 2004.

13. Decreto Legislativo $N^{\circ} 1065$ Modificatoria de la Ley $\mathrm{N}^{\circ} 27314$ del 28 de junio del 2008.

14. MINSA. Norma Técnica "Gestión y manejo de Residuos Sólidos en Establecimientos de Salud y Servicios Médicos de Apoyo, No 096-MINSA/DIGESA V.01 RM No 5542012/Minsa

15. La Corte E. Uso de normas de bioseguridad en el consultorio. Consultado 21-06-2011. Disponible en:http://www.intramed.net/sitios/ mexico/revista_odonto/vol3_pdf_ tapas/Vol_3_5_5.pdf

16. Montaño ML. Manejo de los residuos biológicos infecciosos sólidos, generados por alumnos de la UABC y dentistas ubicados en la zona centro de la ciudad de Mexicali. [Tesis doctoral]. Granada. Facultad de Odontología. Universidad de Granada. 2006.

17. Bustamante BA. Descripción del manejo, almacenamiento y eliminación de los Desechos Sólidos Hospitalarios en la Facultad de Odontología de la Universidad de San Carlos de Guatemala y propuesta para hacerlo congruente con el Acuerdo Gubernativo No 509.2001.[Tesis de titulación] Guatemala. Facultad de Odontología. Universidad de San Carlos de Guatemala. 2006.

18. MINSA. Programa Nacional de Prevención y Control de Infecciones Hospitalarias. La Habana: Folleto, 1998:p. 1-15 